\title{
Decentralized cooperative tracking subject to motion constraints
}

\author{
Lin Wang $^{\text {a }}$, Johan Markdahl ${ }^{\mathrm{b}}$, Zhixin Liu ${ }^{\mathrm{c}}$, Xiaoming $\mathrm{Hu}^{\mathrm{d}}$ \\ ${ }^{a}$ Department of Automation, Shanghai Jiao Tong University, and Key Laboratory of System Control and Information \\ Processing, Ministry of Education, Shanghai, China \\ ${ }^{\mathrm{b}}$ Luxembourg Centre for Systems Biomedicine, University of Luxembourg, Esch-zur-Alzette, Luxembourg \\ ${ }^{\mathrm{c}}$ Key Laboratory of Systems and Control, Academy of Mathematics and Systems Science, CAS, Beijing, China \\ ${ }^{\mathrm{d}}$ Division of Optimization and Systems Theory, Royal Institute of Technology, Stockholm, Sweden
}

\begin{abstract}
This paper addresses the formation control problem, where three agents are tasked with moving an object cooperatively along a desired trajectory while also adjusting its posture to some desired attitudes, i.e. position and attitude tracking. Two decentralized control laws based on locally available information are proposed. The first control law maintains constant inter-agent distances over time, i.e. the formation of agents moves as a single rigid-body. The second control law relaxes this constraint by only maintaining similarity of the agent formation as a polygon in Euclidean space.
\end{abstract}

Key words: Decentralized tracking; formation control; rigidity; motion constraints; attitude control.

\section{Introduction}

Formation control of systems with diverse dynamics and various task requirements has been studied using a number of different approaches to control design. For example, recently, some graphical conditions for distributed formation control were given in [Lin et al., 2014]; Formation control with incomplete information [Jafarian \& Persis, 2015,Liu \& Jiang, 2013], with uncertainty [Dong \& Farrell, 2009], with time-varying formation [Dong et al., 2015, Moya et al., 2016, Turpin, Michael, \& Kumar, 2012] were also investigated. Problems closely related to formation control are consensus, swarm, cooperative target tracking and path following. For an extensive literature review, see [Bai, Arcak, \& Wen, 2011, Oha, Park, \& Ahnb, 2015, Ren \& Beard, 2008, Ren \& Cao, 2011].

Much of the existing research on formation control address the design of decentralized control laws that steer the considered systems to some stable formation. The problem of how to maintain and change the attitude of an already established formation by means of decentralized

Email addresses: wanglin@sjtu.edu.cn (Lin Wang), markdahl@kth.se (Johan Markdahl), lzx@amss.ac.cn (Zhixin Liu), hu@kth.se (Xiaoming Hu). control has generally been given less focus than formation stabilization. A possible application is having multiple robots carry one object in a decentralized fashion. Cooperation in such tasks is both crucial and difficult since any movement caused by one robot will affect the others. If for example the object being carried is rigid, the control should be designed to keep the relative distances between each robot or else they risk dropping or deforming the object.

In this paper, we consider a decentralized control task for three agents to carry an object collectively. The agents are to move the object along a desired trajectory while adjusting its posture to some desired attitude, which should perform three tasks simultaneously: a) The distance between the agents should satisfy some constraints; b) The carried object should follow some desired position path; c) The carried object should follow some desired attitude path.

The motion constraint a) arises from the object being carried by agents cooperatively. The scenario of fixed contact points on the object indicates constant-distance constraints between the agents. Furthermore, in order to change the grasping points to avoid obstacle, we also consider a constraint that allows the structures formed 
by the agents to maintain similarity throughout the evolution. A systematic framework for studying formation motion feasibility was developed in [Tabuada, Pappas, \& Lima, 2005]. Here, in addition to the feasibility analysis, we further investigate the following trajectory and attitude tracking problems.

In order to fulfill the trajectory tracking task b), we add a leader to guide the agents along the desired trajectory. The leader-following approach has been used in various scenarios in the past, where the leader's velocity or acceleration is usually assumed to be known by the followers [Hong, Chen, \& Bushnell, 2008, Liu \& Jiang, 2013,Zhang, Liu, \& Feng, 2015]. In this paper, we design an observer based on measurements of relative positions to calculate the velocity of the leader, and then embed it in the translation input to follow the desired trajectory.

The attitude regulation c) is related to the attitude control problem for a rigid body which has long been a benchmark problem in robotics and related areas, see e.g. [Markley \& Crassidis, 2014, Murray, Li, \& Sastry, 1994, Siciliano \& Khatib, 2008, Wen \& Kreutz-Delgado, 1991]. Much of the early work on attitude control was carried out using minimal representations of orientations such as Euler angles, or global many-to-one representations such as unit quaternions. In this paper, we consider a global parametrization that is in a one-to-one correspondence with the rotation matrix representation of an orientation as in [Bayadi \& Banavar, 2014, Chaturvedi, Sanyal, \& McClamroch, 2011, Markdahl et al., 2017]. Unlike existing work on attitude control where the rigid body is actuated directly, here actuation on the body is mediated through the agents. In comparison with the attitude coordination of multiple rigid bodies in [Bai, Arcak, \& Wen, 2008, Hatanaka et al., 2012, Igarashi et al., 2009] among other works, we focus on the attitude tracking of the object carried by the three agents rather than the attitudes of the agent themselves.Roughly speaking, the formation shaped by the three agents is taken to represent the attitude of the carried object. It is soft rather than hard rigidity.

A closely related research area is the load transport by multiple robots. Experimental results controlling a team of mobile robots in 2-D space were presented in [Antonelli, Arrichiello, \& Chiaverini, 2009], which were designed and implemented in a centralized architecture. Bilateral teleoperation between a single master and multiple cooperative slave robots was considered in [Lee \& Spong, 2005, Rodríguez-Seda et al., 2010], where the slaves tracked their own reference points based on the master robot to perform position tracking and formation control. Decentralized motion and force control were designed for multiple robots to cooperatively transport objects in [Sugar \& Kumar, 2002, Sun \& Mills, 2002, Mellinger et al., 2013, Montemayor \& Wen, 2005], where desired trajectory was assigned to each robot in the motion control, and there was no exchange of information between the robots.

In our approach, decentralized controllers are designed for the agents to estimate the leader's velocity, and there is no pre-designed reference velocity or trajectory specified for each agent to track. Since under many circumstances, the motion control and the force control can be designed separately, in this paper, we focus on the motion control problem and simplify the agent to be single-integrator as in [Cao et al., 2011, Mou, Cao, \& Morse, 2015, Marina, Cao, \& Jayawardhana, 2015, Marina, Jayawardhana, \& Cao, 2016]. Unlike the controllers in the above mentioned references that stabilize a rigid formation to a desired shape, we present a design that keep the shape all the time, and further extend the constant translation and rotation tracking in [Marina, Jayawardhana, \& Cao, 2016] to a time-varying tracking scenario.

\section{Problem Statement}

Assume the rigid object has been carried by the three agents initially, and there are no constraints on the magnitude of the available controlling torques. This enables us to focus on the cooperation issue of the three agents, rather than the force distribution problem. Let $x_{i} \in \mathbb{R}^{3}$ be the position of the $i$-th agent, the dynamic is modeled by a single integrator

$\dot{x}_{i}=u_{i}, \quad i=1,2,3$,

where $u_{i}$ is the control input.

Let $d\left(x_{i}, x_{j}\right)=\left\|x_{i}-x_{j}\right\|^{2}$ denote the distances between agent $i$ and $j$. Two motion constrains are considered, one is the rigid motion constraints that the distance between agents are fixed during the process; the other is the similarity motion constraints that the structures formulated by the three agents are flexible but keep similarity. The similarity motion constraints provide capability for obstacle avoidance and navigation in clustered environments.

Definition 2.1 Rigid motion constraint can be written as $d\left(x_{i}, x_{j}\right)=c_{i, j}$ for $i, j=1,2,3$, where the constants $c_{i, j}>0$ are given by the initial conditions.

Definition 2.2 Similarity motion constraint can be written as $d\left(x_{1}, x_{2}\right)=r_{1} d\left(x_{2}, x_{3}\right)$ and $d\left(x_{2}, x_{3}\right)=$ $r_{2} d\left(x_{3}, x_{1}\right)$, where the constants $r_{1}, r_{2}>0$ are fixed and determined by the initial conditions.

We assume that the three agents are non-collinear, that is the three positions $x_{1}, x_{2}$ and $x_{3}$ uniquely characterize a plane. Since the object should be carried by the three agents all the time, the position and attitude of the object can be completely determined by the states of 
the agents. Therefore, we define the center of the three agents as

$x_{c}=\frac{x_{1}+x_{2}+x_{3}}{3}$,

and define two unit vectors as

$$
\begin{aligned}
& n=\frac{\left(x_{1}-x_{2}\right) \times\left(x_{2}-x_{3}\right)}{\left\|\left(x_{1}-x_{2}\right) \times\left(x_{2}-x_{3}\right)\right\|}, \\
& l=\frac{\left(x_{1}-x_{2}\right)-\left(x_{3}-x_{1}\right)}{\left\|\left(x_{1}-x_{2}\right)-\left(x_{3}-x_{1}\right)\right\|},
\end{aligned}
$$

where $\times$ is the cross product. One can see that $n$ is a normal vector of the plane, and $l=\left(x_{1}-x_{c}\right) /\left\|x_{1}-x_{c}\right\|$ is a vector in the plane.

\section{Motion Constraints}

First, we introduce some notations. Let

$$
\xi=x_{1}-x_{2}, \eta=x_{2}-x_{3}, \zeta=x_{3}-x_{1} .
$$

For a vector $a=\left[a_{1}, a_{2}, a_{3}\right]^{T} \in \mathbb{R}^{3}, S(a) \in \mathbb{R}^{3 \times 3}$ is the skew-symmetric matrix generated by a vector $a=$ $\left[a_{1}, a_{2}, a_{3}\right]^{T} \in \mathbb{R}^{3}$, i.e.

$$
S(a)=\left[\begin{array}{rrr}
0 & -a_{3} & a_{2} \\
a_{3} & 0 & -a_{1} \\
-a_{2} & a_{1} & 0
\end{array}\right]
$$

Note that $S(a) b$, with $b \in \mathbb{R}^{3}$, is an alternative notation for the cross product $a \times b$. This implies, among other things, that $S(a)$ is linear and $S(a) a=0$. Moreover, two properties of cross products are $a^{T}(b \times c)=c^{T}(a \times b)$ and $a \times(b \times c)=b\left(a^{T} c\right)-c\left(a^{T} b\right)$.

\subsection{Rigid motion constraint}

In order to maintain the relative distances $d\left(x_{i}, x_{j}\right)$ at constant values throughout the evolution, $\dot{d}\left(x_{i}, x_{j}\right)=0$ implies that the control of system (1) should satisfy

$$
\begin{aligned}
& \xi^{\tau}\left(u_{1}-u_{2}\right)=0, \\
& \eta^{\tau}\left(u_{2}-u_{3}\right)=0, \\
& \zeta^{\tau}\left(u_{3}-u_{1}\right)=0,
\end{aligned}
$$

i.e. the control law $u=\left[u_{1}^{\tau}, u_{2}^{\tau}, u_{3}^{\tau}\right]^{T}$ should then belong to the null-space of the matrix $M \in \mathbb{R}^{3 \times 9}$ defined by

$$
M=\left[\begin{array}{ccc}
\xi^{T} & -\xi^{T} & 0 \\
0 & \eta^{T} & -\eta^{T} \\
-\zeta^{T} & 0 & \zeta^{T}
\end{array}\right]
$$

Define

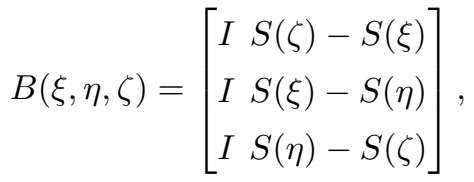

where function $S(\cdot)$ is given by $(6)$. We have $B(\xi, \eta, \zeta) \in$ $\mathbb{R}^{9 \times 6}, \operatorname{rank}(B(\xi, \eta, \zeta))=6$, and $M B(\xi, \eta, \zeta)=0$, which indicates that the columns of $B(\xi, \eta, \zeta)$ form a basis for the kernel of $M$. Therefore, we design a rigid motion controller as

$$
u^{r}=B(\xi, \eta, \zeta)\left[\begin{array}{l}
v \\
w
\end{array}\right]=\left[\begin{array}{l}
v+w \times(\xi-\zeta) \\
v+w \times(\eta-\xi) \\
v+w \times(\zeta-\eta)
\end{array}\right],
$$

where $v, w \in \mathbb{R}^{3}$ are new input signals representing the translational and rotational velocities of the formation. Note that the new inputs $v$ and $w$ can be designed arbitrarily but should be the same for the three agents in order to maintain the rigid motion constraints.

With the rigid motion controller (8), a straightforward calculation yields

$$
\dot{\xi}=\omega \times \xi, \quad \dot{\eta}=\omega \times \eta, \quad \dot{\zeta}=\omega \times \zeta,
$$

where $\omega \triangleq 3 w$ is introduced for notational convenience. The dynamics (9) conserve the norms of $\xi, \eta$ and $\zeta$, as indicated by the fact that $x^{\tau}(\omega \times x)=0$ for all $x \in \mathbb{R}^{3}$.

\subsection{Similarity motion constraint}

Based on the rigid motion controller, we design a similarity motion controller as

$$
u^{s}=\left[\begin{array}{l}
v+w \times(\xi-\zeta)+r(\xi-\zeta) \\
v+w \times(\eta-\xi)+r(\eta-\xi) \\
v+w \times(\zeta-\eta)+r(\zeta-\eta)
\end{array}\right],
$$

where $v, w \in \mathbb{R}^{3}$ are new input signals representing the translational and rotational velocities of the formation, and $r \in \mathbb{R}$ is a new adjustable variable represent the scaling factor.

With the similarity motion controller (10), one has

$$
\dot{\xi}=\omega \times \xi+\mathrm{r} \xi, \dot{\eta}=\omega \times \eta+\mathrm{r} \eta, \dot{\zeta}=\omega \times \zeta+\mathrm{r} \zeta,
$$

where $\omega \triangleq 3 w$ and $\mathrm{r} \triangleq 3 r$ for notational convenience.

Lemma 3.1 The similarity motion controller $u^{s}$ designed by (10) can guarantee the similarity motion constraints. 
Proof: Denote $y=\xi^{\tau} \xi-r_{1} \eta^{\tau} \eta$ and $z=\eta^{\tau} \eta-r_{2} \zeta^{\tau} \zeta$, where $r_{1}, r_{2}$ are given by Definition 2.2. Based on (11), we obtain that $\dot{y}=2 \mathrm{r} y, \dot{z}=2 \mathrm{r} z$. Therefore, $y(t) \equiv 0$ and $z(t) \equiv 0$, in view of $y(0)=0, z(0)=0$ and the uniqueness of the solutions.

Remark 3.1 Each of the three agents is capable of implementing its own control law based on local measurements since $u_{i}^{r}=v+\left[\left(x_{j}-x_{i}\right)+\left(x_{k}-x_{i}\right)\right] \times w$ and $u_{i}^{s}=v+\left[\left(x_{j}-x_{i}\right)+\left(x_{k}-x_{i}\right)\right] \times w+r\left[\left(x_{i}-x_{j}\right)+\left(x_{i}-x_{k}\right)\right]$ are all symmetric with respect to $x_{j}$ and $x_{k}$, i.e. the same $u_{i}^{r}$ and $u_{i}^{s}$ are obtained no matter how agent $i$ chooses to label itself or $j$ and $k$.

Remark 3.2 The variables $v, w$ and $r$ in $u^{r}$ and $u^{s}$ are new input signals, which can be designed arbitrarily but should be the same for the three agents in order to maintain the motion constraints.

\section{Trajectory Following}

No matter if the rigid motion controller (8) or the similarity motion controller (10) is used, the centroid of the three agents given by (2) evolves according to

$\dot{x}_{c}=v$.

Therefore, the translation input $v$ can be designed to fulfill the trajectory tracking task for both controllers simultaneously.

A leader is added to guide the agents. The dynamics of the leader is given by

$\dot{x}_{l}=A x_{l}+b$

where $x_{l}, b \in \mathbb{R}^{3}$, and $A \in \mathbb{R}^{3 \times 3}$ can be any matrix. For example, when $A=-A^{T}$ and $b=-A c$ with $c \in \mathbb{R}^{3}$, the trajectory of $x_{l}$ is a circle centered at $c$, with radius $\left\|x_{l}(0)-c\right\|$.

In order to enable the carried object to track the desired trajectory given by the leader, in view of the internal model principle, the three agents require some structural information concerning the dynamics of the leader. Therefore, we assume that the three agents know $A$ and $b$. Still, the agents do not know the leader's input, since the initial value of the leader's state cannot be measured.

Let $\hat{x}_{i}$ denote the estimation of the leader's state by agent $i$, which is given by

$\dot{\hat{x}}_{i}=A \hat{x}_{i}+P\left(x_{i}-x_{l}\right)+b$,

where $P \in \mathbb{R}^{3 \times 3}$ is yet to be determined. Since the input signal $v$ should be the equal for all three agents, we design

$v=A \sum_{j=1}^{3} \frac{\hat{x}_{j}}{3}+Q \sum_{j=1}^{3} \frac{x_{j}-x_{l}}{3}+b$,

where $Q \in \mathbb{R}^{3 \times 3}$ needs to be determined. Here, agent $i$ can calculate $x_{j}-x_{l}$ in (15) from the relative information $x_{j}-x_{i}$ and $x_{i}-x_{l}$. Alternatively, each agent only calculates its own estimate of the leaders state and communicates it to the other two agents. From (14) and (15), one sees that only relative information is used in the input $v$.

Define

$$
\hat{x}=\sum_{j=1}^{3} \frac{\hat{x}_{j}}{3}, \quad e=x_{c}-x_{l}, \quad \hat{e}=\hat{x}-x_{l} .
$$

Straightforward calculations show that

$$
\dot{e}=Q e+A \hat{e}, \quad \dot{\hat{e}}=P e+A \hat{e} .
$$

The tracking problem has thus been transformed to the problem of finding suitable matrices $P$ and $Q$ such that $\lim _{t \rightarrow \infty} e=0$.

Theorem 4.1 Consider the dynamics (16). Design $P=$ $-K-\left(A+A^{T}\right)$ and $Q=-K-A$, where $K$ is any symmetric, positive definite matrix. Then, $e \rightarrow 0$ and $\hat{e} \rightarrow \mathcal{N}(A)$, where $\mathcal{N}(A)$ is the null space of $A$, as time goes to infinity.

Proof: Introduce the candidate Lyapunov function $V=$ $\frac{1}{2}\|e\|^{2}+\frac{1}{2}\|e-\hat{e}\|^{2}$, with time-derivative $\dot{V}=e^{T} \dot{e}+(e-$ $\hat{e})^{T}(\dot{e}-\dot{\hat{e}})=e^{T} Q e+e^{T} A \hat{e}+e^{T}(Q-P) e-\hat{e}^{T}(Q-P) e=$ $e^{T}\left(Q^{T}+Q-P\right) e-\hat{e}^{T}\left(Q-P-A^{T}\right) e=-e^{T} K e$. Note that $\dot{V} \leq 0$, whereby $e$ and $\hat{e}$ converge to the largest invariant set of points that satisfy $\dot{V}=0$ by LaSalle's invariance principle [Slotine \& Li, 1991]. From $\dot{V}=0$ we obtain $e=0$ and the largest invariant subset of $\{e, \hat{e} \in$ $\left.\mathbb{R}^{3} \mid e=0\right\}$ is $\left\{e, \hat{e} \in \mathbb{R}^{3} \mid e=0, \hat{e} \in \mathcal{N}(A)\right\}$.

System (16) is a simplified model for observer-based tracking problem of leader-follower multi-agent systems. Next, consider a more general tracking error dynamical system

$\dot{e}=s Q e+A \hat{e}, \quad \dot{\hat{e}}=s P e+A \hat{e}$,

where $e, \hat{e} \in \mathbb{R}^{n}$ and $s$ is an unknown parameter bounded below by a known constant $c>0$.

Theorem 4.2 Consider the dynamical system (17). Design $P=-c^{-1} K-A^{T}$ and $Q=-c^{-1} K$, where $K$ is any 
symmetric, positive definite matrix whose smallest eigenvalue $\lambda$ is greater than the spectral radius $\rho(A)$. Then, $e \rightarrow 0$ and $\hat{e} \rightarrow \mathcal{N}(A)$ as time goes to infinity.

Proof: Introduce the candidate Lyapunov function $V=$ $\frac{1}{2}\|e\|^{2}+\frac{1}{2 s}\|e-\hat{e}\|^{2}$, where $s$ is an unknown parameter. Then $\dot{V}=e^{T} \dot{e}+s^{-1}(e-\hat{e})^{T}(\dot{e}-\dot{\hat{e}})=s e^{T} Q e+e^{T} A \hat{e}+$ $e^{T}(Q-P) e-\hat{e}^{T}(Q-P) e=e^{T}((1+s) Q-P) e-\hat{e}^{T}(Q-$ $\left.P-A^{T}\right) e=-e^{T}\left(s c^{-1} K-A^{T}\right) e \leq-e^{T}\left(K-A^{T}\right) e \leq$ $-(\lambda-\rho(A))\|e\|^{2}$. The rest of the proof proceeds as that of Theorem 4.1.

The spectral radius is bounded above by any operator norm. Certain operator norms have closed form expressions, e.g. $\|A\|_{1}$ and $\|A\|_{\infty}$. This makes the proposed control law numerically tractable, one may e.g. set $K=\left(\varepsilon+\max _{j} \sum_{i=1}^{n}\left|a_{i j}\right|\right) I$ for any $\varepsilon>0$.

Remark 4.1 Based on the above analysis, we know that the tracking error will tend to zero, while the estimation error will tends to the null space of the system matrix $A$. Therefore, if $A$ has full rank, then at end the agents can estimate the leader's state precisely.

\section{Attitude Control}

The attitude of any rigid body as expressed in an inertial frame of reference can be described using a rotation axis and a rotation angle by Euler's theorem [Markley $\&$ Crassidis, 2014]. In our case, the vector $n$ that given by the normal of the plane formed by the three agents spans the rotation axis, and the angle of rotation about that axis can be determined by the vector $l$ in (4).

With the notations $\xi, \eta$ and $\zeta$ defined in (5), the vectors $n$ and $l$ can be rewritten as

$n=\frac{\xi \times \eta}{\|\xi \times \eta\|}, \quad l=\frac{\xi-\zeta}{\|\xi-\zeta\|}$

Theorem 5.1 No matter with the rigid motion controller (8) or with the similarity motion controller (10), $n$ and $l$ have the same kinetics

$\left\{\begin{array}{l}\dot{n}=\omega \times n, \\ \dot{l}=\omega \times l,\end{array}\right.$

where $\omega=3 w$.

Proof: The cooperative control protocol (8) enable the three agents to move uniformly as if they formed a single rigid body. Next, consider the similarity motion controller (10).

Define $\phi \triangleq \xi \times \eta$. Based on (11), one has $\dot{\phi}=\dot{\xi} \times$ $\eta+\xi \times \dot{\eta}=(\omega \times \xi+\mathrm{r} \xi) \times \eta+\xi \times(\omega \times \eta+\mathrm{r} \eta)=$ $(\omega \times \xi) \times \eta+\xi \times(\omega \times \eta)+2 \mathrm{r}(\xi \times \eta)=\omega \times \phi+2 \mathrm{r} \phi$. Therefore, with the controller (10), $\|\phi\|^{\prime}=2 \mathrm{r}\|\phi\|$, and correspondingly $\dot{n}=\frac{\dot{\phi}}{\|\phi\|}-\frac{2 \mathrm{r} \phi}{\|\phi\|}=\frac{\omega \times \phi}{\|\phi\|}=\omega \times n$.

Denote $\psi \triangleq \xi-\zeta$. One can obtain $\dot{\psi}=\omega \times \psi+\mathrm{r} \psi$, which indicates $\|\psi\|^{\prime}=\mathrm{r}\|\psi\|$ and furthermore $i=$ $\frac{(\omega \times \psi+\mathrm{r} \psi)\|\psi\|-\mathrm{r}\|\psi\| \psi}{\|\psi\|^{2}}=\frac{\omega \times \psi}{\|\psi\|}=\omega \times l$.

By Theorem 5.1, no matter which of the controller (8) or (10) the agents apply, the attitude of their cooperatively carried object is solely driven by the input signal $\omega$.

Let $n_{d}, l_{d} \in \mathbb{R}^{3}$ be two orthogonal united vectors, which represent the desired attitude. First, in Subsection 5.1, we consider the situation when $n_{d}$ and $l_{d}$ are constant vectors, and design input signal to stabilize the formation of agents to the reference attitude. Then, we consider the situation when $n_{d}$ and $l_{d}$ are time-varying attitude trajectory, and design input signal to fulfill the attitude tracking in Subsection 5.2.

\subsection{Attitude Stabilization}

Assume $n_{d}$ and $l_{d}$ with $\left\|n_{d}\right\|=\left\|l_{d}\right\|=1$ are two orthogonal constant vectors. We first focus on the rotation axis $n$, and present a control law $\omega$ based on relative information that asymptotically drive the three agents to a plane with the desired normal vector $n_{d}$.

Let $P_{a} \subset \mathbb{R}^{3}$ denote a plane with normal $a \in \mathbb{R}^{3}$. Intuitively, the state $\xi$ and $\eta$ dominating the attitude of the three agents can be driven to the reference plane by rotating them about the line of intersection between $P_{n}$ and $P_{n_{d}}$. Therefore, a preliminary input signal $\omega$ is designed as

$\omega=\alpha\left(n \times n_{d}\right)$,

where $\alpha \in \mathbb{R}^{+}$is a scale factor. Extend it as follows

$\omega=\alpha\left(n \times n_{d}\right)+g n$,

where $\alpha \in \mathbb{R}^{+}$is a constant and $g \in \mathbb{R}$ is an arbitrary time- or state-dependent function. Intuitively, the term $\alpha\left(n \times n_{d}\right)$ generates a rotation towards the desired plane $P_{n_{d}}$, and the term $g n$ generates a rotation around the axis spanned by $n$.

Theorem 5.2 With the input signal $\omega$ defined by (21), $n$ will converge to $n_{d}$ from any initial point except $n(0)=$ $-n_{d}$. Furthermore, the rate of convergence is locally exponential.

Proof: Define a Lyapunov function candidate by $V=$ $\frac{1}{2}\left\|n-n_{d}\right\|^{2}=1-n_{d}^{T} n$. Denote $\gamma \triangleq n_{d}^{T} n$. Note that $|\gamma| \leq 1$ and $\dot{\gamma}=n_{d}^{T} \dot{n}=n_{d}^{T}(\omega \times n)=\alpha n_{d}^{T}\left(\left(n \times n_{d}\right) \times n\right)=$ 
$\alpha\left\|n \times n_{d}\right\|^{2} \geq 0$. Then, we have $\dot{V}=-\dot{\gamma}=-\alpha(n \times$ $\left.n_{d}\right)^{T}\left(n \times n_{d}\right)=\alpha n_{d}^{T}\left(n \times\left(n \times n_{d}\right)\right)=\alpha\left[\left(n_{d}^{T} n\right)^{2}-1\right]=$ $-\alpha\left(n_{d}^{T} n+1\right) V=-\alpha(\gamma+1) V \leq 0$. By using LaSalle's invariant principle [Slotine \& Li, 1991], one obtains that the normal vector $n$ will converge to the invariant set $\left\{n_{d},-n_{d}\right\}$. Furthermore, by noting that $\gamma$ is increasing, one has $n$ converges to $n_{d}$ exponentially for all initial states except $n(0)=-n_{d}$.

Based on the above analysis, we know that (i) the term $\alpha\left(n \times n_{d}\right)$ contributes to the convergence to the desired plane $P_{n_{d}}$; (ii) the term $g n$ does not contribute nor interfere with said convergence but can be used to regulate the rotation of the configuration about $n$. Next, we will further make use of the second term by designing $g$ such that the configuration formed by all agents can reach a desired attitude characterized by $n_{d}$ and $l_{d}$. To achieve this goal we choose

$$
g=\beta n^{T}\left(l \times l_{d}\right),
$$

where $\beta \in \mathbb{R}^{+}$and $l_{d}$ is a reference value for $l$ defined in (4). The input signal $\omega$ becomes

$\omega=\alpha\left(n \times n_{d}\right)+\beta\left(n^{T}\left(l \times l_{d}\right)\right) n$.

Theorem 5.3 Assume the input signal $\omega$ is given by (23) and $n(0) \neq-n_{d}$. Then there are two equilibria $l=$ $\pm l_{d}$ on the invariant set $P_{n_{d}}$, where $l=-l_{d}$ is unstable while $l=l_{d}$ is locally exponentially stable.

Proof: Theorem 5.2 has presented that $n$ will exponentially converge to $n_{d}$ from any initial point except $n(0)=$ $-n_{d}$, no matter which kind of function $g$ is chosen. Moreover, for Lyapunov function $V=\frac{1}{2}\left\|n-n_{d}\right\|^{2}=1-n_{d}^{T} n$, we have $\dot{V}=-\alpha\left(n \times n_{d}\right)^{T}\left(n \times n_{d}\right)$.

Introduce a new Lyapunov candidate function $U=\frac{1}{2} \| l-$ $l_{d} \|^{2}=1-l_{d}^{T} l$. The time derivative of $U$ is $\dot{U}=-l_{d}^{T} \dot{l}=$ $-l_{d}^{T}(\omega \times l)=\omega^{T}\left(l_{d} \times l\right)=\left(\alpha\left(n \times n_{d}\right)+\beta\left(n^{T}(l \times\right.\right.$ $\left.\left.\left.l_{d}\right)\right) n\right)^{T}\left(l_{d} \times l\right)=\alpha\left(n \times n_{d}\right)^{T}\left(l_{d} \times l\right)-\beta\left(n^{T}\left(l \times l_{d}\right)\right)^{2}$, which is sign indefinite.

Form a composite Lyapunov-candidate-function $W$ of $V$ and $U$ by $W=\kappa V+U, \kappa \in \mathbb{R}^{+}$. One has $\dot{W}=\kappa \dot{V}+\dot{U}=$ $-\kappa \alpha\left\|n \times n_{d}\right\|^{2}+\alpha\left(n \times n_{d}\right)^{T}\left(l_{d} \times l\right)-\beta\left(n^{T}\left(l \times l_{d}\right)\right)^{2} \leq$ $-\kappa \alpha\left\|n \times n_{d}\right\|^{2}+\alpha\left\|n \times n_{d}\right\|\left\|l \times l_{d}\right\|-\beta\left\|l \times l_{d}\right\|^{2} \cos ^{2} \theta \triangleq$ $-z^{T} M z$, where $\theta$ is the angle between $n$ and $l \times l_{d}, z \triangleq$ $\left[\left\|n \times n_{d}\right\|,\left\|l \times l_{d}\right\|\right]^{T}$, and

$M \triangleq\left[\begin{array}{ll}\kappa \alpha & -\frac{\alpha}{2} \\ -\frac{\alpha}{2} & \beta \cos ^{2} \theta\end{array}\right]$

From Theorem 5.2, we know that $n \rightarrow n_{d}$ exponentially. Therefore, there exist a finite time $T$ after which $\cos ^{2} \theta \geq$ $\frac{1}{2}$, where we assume $\theta=0$ in the case of $l=l_{d}$. Choose parameter $\kappa$ for example $\kappa=\frac{2 \alpha}{\beta}$, such that the matrix $M$ is positive definite. Then, we obtain that $\dot{W} \leq 0$ for $t>T$.

For $t>T, W$ is monotonic and bounded from below by 0 . Therefore, $W$ has a finite limit as $t \rightarrow \infty$. Since the function $\dot{W}$ is uniformly continuous, we have $\lim _{t \rightarrow \infty} \dot{W}=0$ by Barbalat's lemma [Slotine \& Li, 1991]. Since $\lim _{t \rightarrow \infty} n=$ $n_{d}$ from Theorem 5.2, we have $\lim _{t \rightarrow \infty} n_{d}^{T} l=0$ in view of $n^{T} l=0$, and $\lim _{t \rightarrow \infty} \dot{W}=-\lim _{t \rightarrow \infty} \beta\left(n_{d}^{T}\left(l \times l_{d}\right)\right)^{2}$. Therefore, $\lim _{t \rightarrow \infty} l= \pm l_{d}$.

The instability of $(n, l)=\left(n_{d},-l_{d}\right)$ follows from letting $n(0)=n_{d}$. Then $n=n_{d}$ for all the time, and $\dot{U}=$ $-\beta\left(n^{T}\left(l \times l_{d}\right)\right)^{2}=-\beta\left\|l \times l_{d}\right\|^{2}$, which is negative for all $l \neq \pm l_{d}$. The point $l=-l_{d}$ is a maximizer of $U$ and no function can converge to its maximum value with a strictly negative derivative. Hence $l=-l_{d}$ is unstable.

The stability of $(n, l)=\left(n_{d}, l_{d}\right)$ follows from $\dot{W}<0$ for a hollow neighborhood of $\left(n_{d}, l_{d}\right)$. Next, we show the locally exponential rate of convergence of $W$. Based on the previous analysis, we know that there exist a finite time $T$ after which $\dot{W} \leq-\kappa \alpha\left\|n \times n_{d}\right\|^{2}+\alpha\left\|n \times n_{d}\right\| \| l \times$ $l_{d}\left\|-\frac{\beta}{2}\right\| l \times l_{d}\left\|^{2}=-\kappa \alpha\right\| n \times n_{d}\left\|^{2}+\alpha \sqrt{\frac{2}{\beta}}\right\| n \times n_{d}\left\|\sqrt{\frac{\beta}{2}}\right\| l \times$ $l_{d}\left\|-\frac{\beta}{2}\right\| l \times l_{d}\left\|^{2} \leq\left(-\kappa \alpha+\frac{\alpha^{2}}{\beta}\right)\right\| n \times n_{d}\left\|^{2}-\frac{\beta}{4}\right\| l \times l_{d} \|^{2}=$ $-\left(\kappa \alpha-\frac{\alpha^{2}}{\beta}\right)\left(1+n_{d}^{T} n\right) V-\frac{\beta}{4}\left(1+l_{d}^{T} l\right) U$. Choose parameter $\kappa$ such that $\kappa \alpha-\frac{\alpha^{2}}{\beta}>0$ (for example $\kappa=\frac{2 \alpha}{\beta}$ ). In view of $\left|n_{d}^{T} n\right| \leq 1$ and $\left|l_{d}^{T} l\right| \leq 1$, we have

$$
\dot{W} \leq-\min \left\{\left(\alpha-\frac{\alpha^{2}}{\kappa \beta}\right)\left(1+n_{d}^{T} n\right), \frac{\beta}{4}\left(1+l_{d}^{T} l\right)\right\} W
$$

with a negative right hand side for a neighborhood of $\left(n_{d}, l_{d}\right)$, establishes the locally exponential convergence rate of $W$.

\subsection{Attitude tracking}

In this subsection, we assume the desired orientation is a time-varying trajectory, i.e. $n_{d}$ and $l_{d}$ are time varying. The control objective is to enable the carried object track the desired attitude path by having $n$ track $n_{d}$, and $l$ $\operatorname{track} l_{d}$.

Introduce

$m=\frac{n \times l}{\|n \times l\|}, \quad m_{d}=\frac{n_{d} \times l_{d}}{\left\|n_{d} \times l_{d}\right\|}$, 
and define a body frame and a desired body frame for the formation of agents as

$R=[l, m, n], \quad R_{d}=\left[l_{d}, m_{d}, n_{d}\right]$.

Note that $R$ evolves with the state of the three agents, and $R, R_{d} \in S O(3)$ with $S O(3)=\left\{R \in \mathbb{R}^{3 \times 3} \mid R R^{T}=\right.$ $\left.R^{T} R=I, \operatorname{det} R=1\right\}$.

Based on Theorem 5.1, we known that the rigid motion controller (8) and the similarity motion controller (10) enable the same kinetics for $n$ and $l$, that $\dot{n}=\omega \times n$, $i=\omega \times l$, which further enables $\dot{m}=\omega \times m$. Therefore, we obtain that

$\dot{R}=S(\omega) R$

If we can design rotational signal $\omega$ such that $R-R_{d} \rightarrow 0$, then the attitude tracking problem will be solved.

Note that $R_{d} R_{d}^{T}=I$. Therefore, we have $\dot{R}_{d} R_{d}^{T}+$ $R_{d} \dot{R}_{d}^{T}=0$, or $\dot{R}_{d} R_{d}^{T}=-\left(\dot{R}_{d} R_{d}^{T}\right)^{T}$. That is $\dot{R}_{d} R_{d}^{T}$ is a skew-symmetric matrix, and moreover $\dot{R}_{d}=$ $-\left(\dot{R}_{d} R_{d}^{T}\right)^{T} R_{d}$. Let $\omega_{d}$ be the unique vector such that $S\left(\omega_{d}\right)=\dot{R}_{d} R_{d}^{T}$. Then, we have $\dot{R}_{d}=-S\left(\omega_{d}\right) R_{d}$.

Define the error $E=R_{d}^{T} R$, then, the attitude tracking $R-R_{d} \rightarrow 0$ is equivalent to the attitude stabilization $E \rightarrow I$. The derivative of $E$ is $\dot{E}=\dot{R}_{d}^{T} R+R_{d}^{T} \dot{R}=-R_{d}^{T} S\left(\omega_{d}\right) R+R_{d}^{T} S(\omega) R=$ $R_{d}^{T}\left(S\left(\omega-\omega_{d}\right)\right) R_{d} E=S\left(R_{d}^{T}\left(\omega-\omega_{d}\right)\right) E$, where we use the property $M S(y) M^{T}=S(M y)$ for any $M \in S O(3)$.

Define $I_{1}=[1,0,0]^{T}, I_{3}=[0,0,1]^{T}$, and $\omega_{I}=\alpha(n \times$ $\left.I_{3}\right)+\beta\left(n^{T}\left(l \times I_{1}\right)\right) n$. From Theorem 5.3 , we know that $\omega_{I}$ can drive the system $\dot{Q}=S\left(\omega_{I}\right) Q$ to $I$ almost globally for all $Q \in S O(3)$. Therefore, we can use

$\omega_{t}=\omega_{d}+R_{d} \omega_{I}$

to fulfill the attitude tracking task.

\section{Conclusions}

We have proposed two decentralized control laws for three individual agents to cooperatively translate and rotate a carried object with rigid and similarity motion constraints. A leader is introduced to guide the agents' translation. The trajectory tracking part of the proposed control signal includes a distributed observer of the leader's position, which has been shown to guarantee asymptotical stability of the tracking error and stability of the observer error. The attitude stabilization is solved by design rotational axes which consist of the normal vector of the agents' plane and the intersection line of the agents' plane with the desired plane. By treating the attitude of the formation shaped by the three agents as a rigid body, the attitude tracking is solved based on the attitude stabilization. Future work will focus on extension to the case of distributed tracking for $n$ agents subject to motion constraints; and consideration of load transport in a more realistic manner.

\section{Acknowledgements}

This work was supported by the National Natural Science Foundation of China under Grant Nos. 61473189, 61621003, 61773255 and 11688101, the Natural Science Foundation of Shanghai (No. 17ZR1445200), and the Swedish Research Council.

\section{References}

[Antonelli, Arrichiello, \& Chiaverini, 2009] Antonelli, G., Arrichiello, F., \& Chiaverini, S. (2009) Experiments of formation control with multirobot systems using the null-space-based behavioral control, IEEE Transactions on Control Systems Technology, 17(5), 1173-1182.

[Bai, Arcak, \& Wen, 2008] Bai, H., Arcak, M., \& Wen, J.T. (2008). Rigid body attitude coordination without inertial frame information. Automatica, 44, 3170-3175.

[Bai, Arcak, \& Wen, 2011] Bai, H., Arcak, M., \& Wen, J. (2011). Cooperative Control Design: A Systematic, Passivity-Based Approach. Springer.

[Bayadi \& Banavar, 2014] Bayadi, R., \& Banavar, R.N. (2014). Almost global attitude stabilization of a rigid body for both internal and external actuation schemes. European Journal of Control. 20, 45-54.

[Cao et al., 2011] Cao, M., Morse, A.S., Yu, C., Anderson, B.D.O., \& Das gupta, S. (2011). Maintaining a directed, triangular formation of mobile autonomous agents. Communications in Information and Systems, 11(1), 1-16.

[Chaturvedi, Sanyal, \& McClamroch, 2011] Chaturvedi, N. A., Sanyal, A.K., \& McClamroch, N. H. (2011). Rigidbody attitude control: Using rotational matrices for continuous, singularity-free control laws. IEEE Control Systems Magazine, 30-51.

[Dong \& Farrell, 2009] Dong, W., \& Farrell, J. A. (2009). Decentralized cooperative control of multiple nonholonomic dynamic systems with uncertainty. Automatica, 45, 706-710.

[Dong et al., 2015] Dong, X. W., Yu, B. C., Shi, Z. Y., \& Zhong, Y. S. (2015). Time-varying formation control for unmanned aerial vehicles: Theories and applications. IEEE Transactions on control systems technology, 23(1), 340-348.

[Jafarian \& Persis, 2015] Jafarian, M., \& Persis, C. D. (2015). Formation control using binary information. Automatica, 53, $125-135$.

[Hatanaka et al., 2012] Hatanaka, T., Igarashi, Y., Fujita, M., \& Spong, M.W. (2012). Passivity-based pose synchronization in three dimensions. IEEE Transactions on Automatic Control, $57(2), 360-375$.

[Hong, Chen, \& Bushnell, 2008] Hong, Y., Chen, G., \& Bushnell, L. (2008). Distributed observers design for leader-following control of multi-agent networks. Automatica, 44(3), 846-850. 
[Igarashi et al., 2009] Igarashi, Y., Hatanaka, T., Fujita, M., \& Spong, M.W. (2009). Passivity-Based Attitude Synchronization in $\mathrm{SE}(3)$. IEEE Trans. Control Systems Technology, 17(5), 1119-1134.

[Lee \& Spong, 2005] Lee, D., \& Spong, M.W. (2005). Bilateral teleoperation of multiple cooperative robots over delayed communication networks: Theory. International Conference on Robotics and Automation, 360-365.

[Lee, 2012] Lee, T. (2012). Exponential stability of an attitude tracking control system on $S O(3)$ for large-angle rotational maneuvers. Systems \& Control Letters, 61(1), 231-237.

[Lin et al., 2014] Lin, Z. Y., Wang, L. L., Han, Z. M., \& Fu, M. Y. (2014). Distributed formation control of multi-agent systems using complex Laplacian. IEEE Trans. on Automatic Control, 59(7), 1765-1177.

[Liu \& Jiang, 2013] Liu, T. F., \& Jiang, Z. P. (2013). Distributed formation control of nonholonomic mobile robots without global position measurements. Automatica, 49, 592-600.

[Marina, Cao, \& Jayawardhana, 2015] Marina, H.G., Cao, M., \& Jayawardhana, B. (2015). Controlling rigid formations of mobile agents under inconsistent measurements. IEEE Transactions on Robotics, 31(1), 31-39.

[Marina, Jayawardhana, \& Cao, 2016] Marina, H.G., Jayawardhana, B., \& Cao, M. (2016). Distributed rotational and translational maneuvering of rigid formations and their applications. IEEE Transactions on Robotics, 32(3), 684-697.

[Markdahl et al., 2017] (2017). Markdahl, J., Hoppe, J., Wang, L., Hu, X. (2017). A geodesic control law to decouple the full and reduced attitude. Systems and Control Letters, 102, $32-41$.

[Markley \& Crassidis, 2014] Markley, F.L. \& Crassidis, J.L. (2014). Fundamentals of Spacecraft Attitude Determination and Control, Springer New York.

[Mellinger et al., 2013] Mellinger, D., Shomin, M., Michael, N., Kumar, V. (2013) Cooperative grasping and transport using multiple quadrotors. In: Martinoli A. et al. (eds) Distributed Autonomous Robotic Systems, Springer, Berlin, Heidelberg.

[Montemayor \& Wen, 2005] Montemayor G., \& Wen, J.T. (2005). Decentralized collaborative load transport by multiple robots, IEEE International Conference on Robotics and Automation, Spain, 372-377.

[Mou, Cao, \& Morse, 2015] Mou, S., Cao, M., Morse, A.S. (2015). Target-point formation control. Automatica, 61, 113-118.

[Moya et al., 2016] Moya, V., Espinosa, V., Chávez, D., Leica, P., \& Camacho, O. (2016). Trajectory tracking for quadcopter's formation with two control strategies. IEEE Ecuador Technical Chapters Meeting, 1-6.

[Murray, Li, \& Sastry, 1994] Murray, R. M., Li, Z. X., \& Sastry, S. S. (1994). A Mathematical Introduction to Robotic Manipulation, CRC Press.

[Oha, Park, \& Ahnb, 2015] Oha, K. K., Park, M. C., \& Ahnb, H. S. (2015). A survey of multi-agent formation control. Automatica, 53, 424-440.

[Ren \& Beard, 2008] Ren, W., \& Beard, R. W. (2008). Distributed Consensus in Multi-Vehicle Cooperative Control. Springer-Verlag.

[Ren \& Cao, 2011] Ren, W., \& Cao, Y. (2011). Distributed Coordination of Multi-agent Networks: Emergent Problems, Models, and Issues. Springer.

[Rodríguez-Seda et al., 2010] Rodríguez-Seda, E.J., Troy, J.J., Erignac, C.A., Murray, P., Stipanović, D. M., \& Spong, M.W. (2010). Bilateral teleoperation of multiple mobile agents:
Coordinated motion and collision avoidance. IEEE Trans. Control Systems Technology. 18(4), 984-992.

[Siciliano \& Khatib, 2008] Siciliano, B., \& Khatib, O. (2008). Handbook of Robotics, Springer-Verlag.

[Slotine \& Li, 1991] Slotine, J. E., \& Li, W. (1991). Applied Nonlinear Control. Prentice Hall: New Jersey.

[Sugar \& Kumar, 2002] Sugar, T.G., \& Kumar, V. (2002). Control of cooperating mobile manipulators. IEEE Trans. Robotics and Automation, 18(1), 94-103, 2002.

[Sun \& Mills, 2002] Sun, D., \& Mills, J.K. (2002). Manipulating rigid payloads with multiple robots using compliant grippers. IEEE Trans. Mechatronics, 7(1), 23-34.

[Tabuada, Pappas, \& Lima, 2005] Tabuada, P., Pappas, G.J., \& Lima, P. (2005). Motion feasibility of multi-agent formations. IEEE Transactions on Robotics, 21(3), 387-392.

[Turpin, Michael, \& Kumar, 2012] Turpin, M., Michael,N., \& Kumar,V. (2012). Trajectory design and control for aggressive formation flight with quadrotors. Auton Robot, 33, 143-156.

[Wen \& Kreutz-Delgado, 1991] Wen, J., \& Kreutz-Delgado, K. (1991). The attitude control problem. IEEE Transactions on Automatic Control, 36(10), 1148-1162.

[Zhang, Liu, \& Feng, 2015] Zhang, X. F., Liu, L., \& Feng, G. (2015). Leader-follower consensus of time-varying nonlinear multi-agent systems. Automatica, 52, 8-14. 